\section{Nuevos valores para el espermiograma OMS 2010}

\section{Updated reference values for sperm counts (WHO 2010)}

\section{Sr. Editor:}

En los últimos años, en Latinoamérica así como en el resto del mundo, las consultas por infertilidad han experimentado un incremento notable. El análisis seminal continúa siendo la herramienta básica de rutina que brinda la mejor información para evaluar la calidad reproductiva del varón. Este examen permite estimar la severidad del factor masculino en la infertilidad y establecer las posibles estrategias terapéuticas para la pareja.

En la determinación de los parámetros seminales, existe un grado de error analítico donde el espermiograma pierde por completo su utilidad clínica si no se realiza bajo estrictas normas de control $^{1}$. En respuesta a la gran necesidad de estandarizar o sistematizar los procedimientos asociados al análisis seminal, la Organización Mundial de la Salud (OMS) ha publicado sucesivas ediciones del "Manual para el Examen del Semen Humano y la Interacción Moco Semen (1980, 1987, 1992 y 1999)", las cuales han servido de guía para los laboratorios de andrología clínica. Sumando esfuerzos para unificar conceptos, la Sociedad Europea de Reproducción Humana y Embriología (ESHRE) y su Grupo de Interés en Andrología (SIGA) junto con la Asociación Nórdica de Andrología (NAFA), organizan desde 1994 un Curso Teórico-Práctico de Análisis Básico de Semen, el cual se ha reproducido en distintos países europeos. Esta herramienta docente junto a la publicación de un Manual (2002) han contribuido a disminuir significativamente las diferencias entre centros y permitido establecer programas confiables de control de calidad ${ }^{2}$.

Durante el International Congress of Andrology realizado en marzo de 2010 en Barcelona, España, y el Primer taller del Programa Latinoamericano para la Estandarización del Análisis Seminal (PLEAS) realizado en mayo de 2010 en Santiago de Chile, la OMS presentó el nuevo Manual "WHO Laboratory Manual for the examination and processing of human semen" (5ta edición), disponible en el sitio web http://whqlibdoc.who. int/publications/2010/9789241547789_eng.pdf e impreso. La principal diferencia de este Manual con respecto a los anteriores y otros de su tipo es que, por primera vez, los valores que se proponen han sido obtenidos de varones cuya pareja ha logrado el embarazo durante los últimos 12 meses, por lo que son considerados "fértiles". Se analizaron los datos de casi 1.900 pacientes en

Tabla. Valores de referencia (1999) y los nuevos (2010) del límite de referencia inferior (LRI) en espermiograma; entre paréntesis se muestra el intervalo de confianza del $95 \%$

\begin{tabular}{|c|c|c|}
\hline & $1999,4^{\text {ta }}$ edición ${ }^{3}$ & 2010, $5^{\text {ta }}$ edición ${ }^{4}$ \\
\hline & Valor de referencia & Límite inferior de referencia, LRL \\
\hline Licuefacción & Total a los $60 \mathrm{~min}$ & Total a los $60 \mathrm{~min}$ \\
\hline $\mathrm{pH}$ & $7,2-7,8$ & $\geq 7,2$ \\
\hline volumen & $2,0 \mathrm{~mL}$ & $1,5 \mathrm{~mL}(1,4-1,7)$ \\
\hline Concentración espermática & $20 \times 10^{6} / \mathrm{mL}$ & $15 \times 10^{6} / \mathrm{mL}(12-15)$ \\
\hline Concentración total & $40 \times 10^{6}$ & $39 \times 10^{6}(33-46)$ \\
\hline Motilidad total (progresivos + no progresivos) & No detallada & $40 \%(38-42)$ \\
\hline Motilidad progresiva & $50 \%$ & $32 \%(31-34)$ \\
\hline Viabilidad & $75 \%$ & $58 \%(55-63)$ \\
\hline Formas normales & $15 \%$ & $4 \%(3-4)$ \\
\hline Leucocitos & $<1 \times 10^{6} / \mathrm{mL}$ & $<1 \times 10^{6} / \mathrm{mL}$ \\
\hline Mar test & $<50 \%$ esp. unidos a partículas & $<50 \%$ esp. unidos a partículas \\
\hline "Immunobeads" & $<50 \%$ esp. unidos a partículas & $<50 \%$ esp. unidos a partículas \\
\hline
\end{tabular}


8 países y 3 continentes y se acordó cambiar el concepto de "valor de referencia" por el de "límite de referencia inferior" (LRI, al $5^{\circ}$ percentil). Este límite separa donde se concentra más de $95 \%$ de la población fértil, o sea por arriba del LRI está el 95\% de los pacientes fértiles. Los valores que caigan por encima del LRI no garantizan la fecundidad y posterior embarazo, pero amplían sus posibilidades. Este nuevo marco conceptual, sumado a cambios en la metodología sugerida, hace necesario una buena interpretación y amplia difusión del mismo entre Tecnólogos Médicos y Médicos. Presentamos una Tabla con el LRI; sin embargo, la OMS recalca que estos valores son sólo una guía y que cada laboratorio, dependiendo de su realidad geográfica, debe hacer esfuerzos para obtener sus propios valores.

Luis Sarabia ${ }^{1}$, María José Munuce ${ }^{2}$ ${ }^{1}$ Presidente del Programa Latinoamericano para la Estandarización del Análisis Seminal (PLEAS), Universidad de Chile. Santiago de Chile.

${ }^{2}$ Miembro de PLEAS, Universidad Nacional del Rosario, Rosario, Argentina.

\section{Referencias}

1. Castilla J, Álvarez C, Aguilar J, González-Varea C, Zalvo M, Martínez L. Influence of analytical and biological variation on the clinical interpretation of seminal parameters. Hum Reprod 2006; 21: 847-51.

2. Björndahl L, Barratt CL, Fraser LR, Kvist U, Mortimer D. ESHRE basic semen analysis courses 1995-1999: immediate beneficial effects of standardized training. Hum Reprod 2002; 17: 1299-305.

3. World Health Organization. WHO Laboratory Manual for the examination of human semen and spermcervical mucus interaction. Cambridge: Cambridge University Fourth Edition (1999).

4. World Health Organization. "WHO Laboratory Manual for the examination and processing of human semen" Cambridge: Cambridge University. Fifth Edition (2010). http://whqlibdoc.who.int/publications/ 2010/9789241547789_eng.pdf.

Correspondencia a:

Profesor Luis Sarabia.

E-mail: Isarabia@med.uchile.cl 